\title{
Nitrofurantoin and Fosfomycin Susceptibility Among Outpatient Uropathogens in a Tertiary Care Center in Southern Thailand
}

Tharntip Sangsuwan, M.D. ${ }^{1}$, Silom Jamulitrat, M.D. ${ }^{1}$, Manthana Phengmak, M.Sc. ${ }^{2}$

'Department of Family Medicine and Preventive Medicine, ${ }^{2}$ Department of Pathology, Faculty of Medicine, Prince of Songkla University, Hat Yai, Songkhla 90110, Thailand.

Received 9 August 2017 • Accepted 20 February 2018 • Published online 24 May 2018

\section{Abstract:}

Objective: To document susceptibility to fosfomycin and nitrofurantoin among uropathogens isolated from the Out Patient Department and Emergency Room of Songklanagarind Hospital.

Material and Methods: From October 2011 to May 2012, bacteria isolated from urine specimens of outpatients with pyuria were evaluated for susceptibility to nitrofurantoin and fosfomycin. The medical records of the patients were reviewed for uropathogens and other antimicrobial susceptibilities. The urinary tract infections (UTIs) were classified into complicated and uncomplicated. Factors associated with fosfomycin and nitrofurantoin susceptibilities were also studied.

Results: The study included 324 patients with positive urine cultures. Complicated UTIs and uncomplicated UTIs were found in $72.8 \%$ and $27.2 \%$, respectively. The most common causative uropathogen was Escherichia coli (E. coli) (71.9\%) followed by Klebsiella pneumoniae (16.7\%). The susceptibility of E. coli to fosfomycin was $98.7 \%$, and the susceptibilities of E. coli, K. pneumoniae, and overall pathogens to nitrofurantoin were $91.0 \%, 24.1 \%$ and $74.1 \%$ respectively.

Conclusion: The uropathogens in Songklanagarind Hospital have higher susceptibilities to fosfomycin than to nitrofurantoin.

Keywords: fosfomycin, nitrofurantoin, susceptibility, uropathogens, UTIs 


\section{Introduction}

Urinary tract infections (UTIs) are among the most prevalent infectious diseases, with a substantial financial burden on society. Normally fluoroquinolones are recommended for treatment of uncomplicated UTIs by the Infectious Diseases Society of America (IDSA), ${ }^{1}$ with trimethoprim (co-trimoxazole) as first-line empirical therapy only in communities with resistance rates of uropathogens to trimethoprim of $\leq 10.0-20.0 \%$.

In Songklanagarind Hospital, the major tertiary care and referral center in southern Thailand, the three most common causative uropathogens in recent years have been Escherichia coli (E. coli) (51.2\%), Klebsiella pneumonia (K. pneumoniae) (8.1\%) and Staphylococcus aureus (S. aureus) $(6.9 \%){ }^{2} \mathrm{~A}$ recent study ${ }^{2}$ found that the susceptibilities of overall uropathogens to cotrimoxazole and norfloxacin were $48.3 \%$ and $59.9 \%$ respectively.

Given the increasing prevalence of resistance to antibiotics commonly used to treat cystitis in recent years, additional therapeutic choices were needed, and fosfomycin trometamol and nitrofurantoin were tried as alternative drug. Previous studies during 1999-2012 in many countries such as Russia, ${ }^{3}$ Greece, ${ }^{4}$ Sweden, ${ }^{5}$ and Rwanda ${ }^{6}$ found that E. coli was the most common pathogen causing UTIs, and $97.0 \%, 93.0 \%, 85.3 \%, 62.1 \%$, respectively, of the isolated pathogens in these studies were susceptible to nitrofurantoin. In Greece ${ }^{4}$ and Rwanda, ${ }^{6} 99.0 \%$ and $92.3 \%$, respectively, of the isolated pathogens were susceptible to fosfomycin.

Reliable knowledge concerning antimicrobial susceptibility is essential for appropriate therapy. The purpose of this study was to determine the susceptibility of the most important uropathogens to fosfomycin and nitrofurantoin.

\section{Material and Methods}

\section{Setting}

Songklanagarind Hospital is 860-bed tertiary care facility serving as a medical school with residency training, and referral center for the southern part of Thailand. On average we see $2,200-2,400$ outpatients per day.

\section{Patient identification}

From $1^{\text {st }}$ October 2011 to $10^{\text {th }}$ May 2012, urine specimens were prospectively collected from patients who came to an Out Patient Department (OPD): Primary Care Unit, Pediatric Clinic, Surgery Clinic, Internal Medicine Clinic, Obstetrics and Gynecology Clinic (OB-GYN), Antenatal Care Clinic, General Practice Clinic, Ear Nose Throat Clinic (ENT), Eye Clinic, Psychology Clinic or Emergency Room (ER). Urine analysis and urine culture were performed. Specimens with a single bacterial species isolated and urine analysis showing pyuria were included in the study. Patient specimens with the same hospital number $(\mathrm{HN})$ were excluded. Definitions of complicated or uncomplicated UTIS were based on the factors which triggered the infection, i.e. a complicated UTIs was an infection associated with a condition, structural abnormality or functional abnormality of the voiding system, obstructive uropathy, urinary stones, retained Foley catheter within the last 12 months, history of urinary tract surgery or pregnancy. Uncomplicated adult UTIs were seen mostly in women without structural or functional abnormalities within the urinary tract.

The study protocol was approved by the Ethics Committees of the Faculty of Medicine, Prince of Songkla University (EC: 55-010-09-1-3). Because of the observational nature of the study, written informed consent was not required.

\section{Data collection}

The data were recorded in a data extraction form which was based on the number of urine culture specimens collected from the patients presenting at the OPD or the ER for whom UTIs was considered a possibility based on their clinical backgrounds and a positive urine culture. Other variables collected for analysis included antimicrobial 
prescribed for UTIs treatment, clinical feature suggesting complicated UTIs (male gender, diabetes mellitus status, pregnancy, structural or functional urinary tract abnormalities, retained urinary catheter, recent urological operation or presence of urinary calculi), history of previous UTIs in the last 12 months, history of previous hospitalization in the last 12 months and history of previous antibiotic usage in the last 6 months (Figure 1).

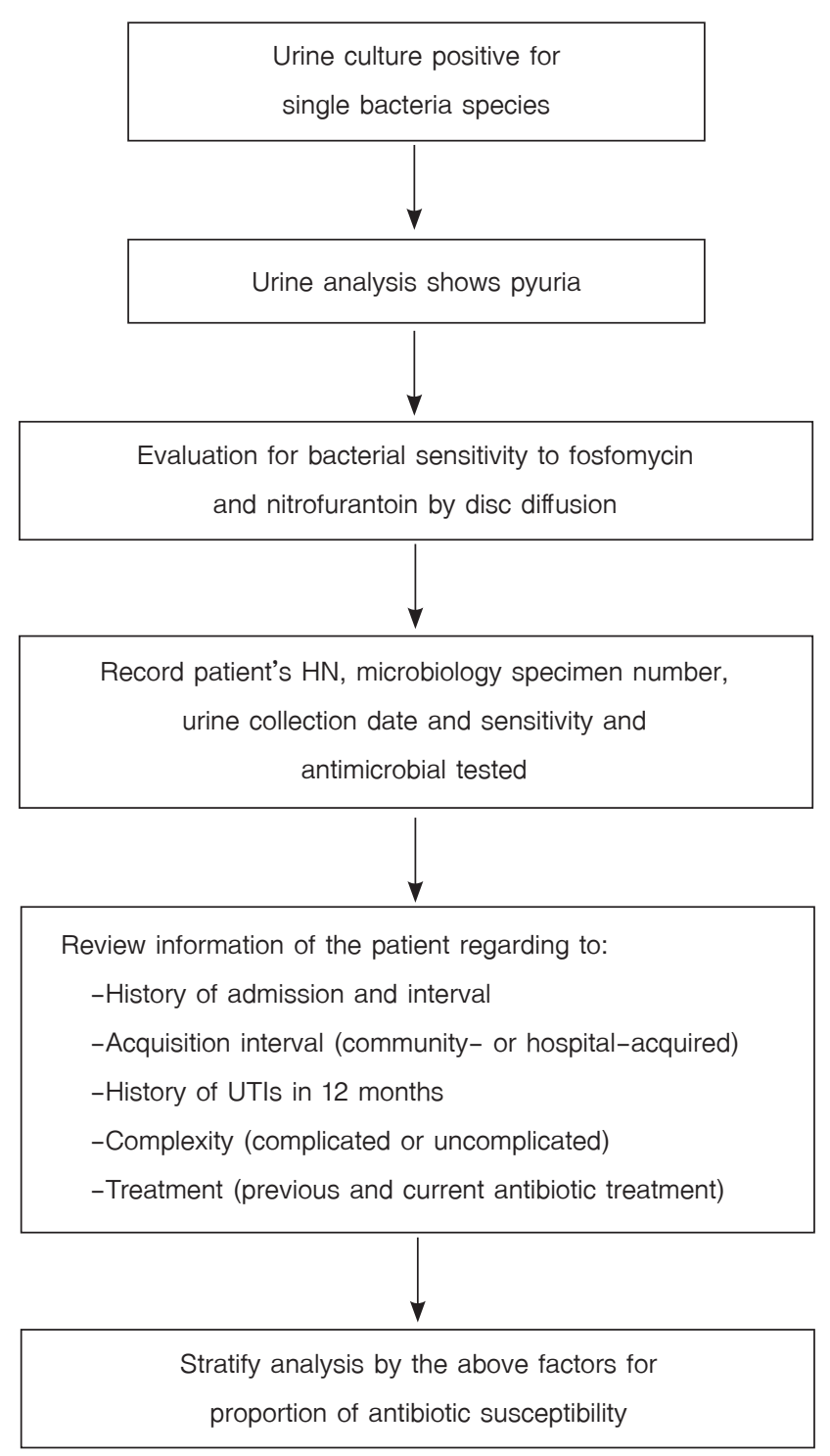

Figure 1 Material and methods

\section{Variables}

Variables collected were sex, age, history of admission in the last 12 months, interval between the latest admission and this visit, history of previous UTIs in 12 months, complicated UTIs, urine collection methods, urine cultures (identification of organism), treatment (previous and current antibiotic treatment), antimicrobial susceptibility test results, inhibition zone of fosfomycin and nitrofurantoin

\section{Microbiology \\ Urine collection and processing}

It is the usual practice in our institution that a patient with clinical signs or symptoms indicating the possibility of UTIs is further investigated by quantitative urine culture. A urine sample is collected from the patient by means of the midstream clean-catch technique or catheterization and sent to the microbiology laboratory immediately for culture and identification. At the microbiology laboratory, the urine is placed on a standard loop (a 1 microliter loop for midstream urine, a 10-microliter loop for a catheter-obtained sample) and inoculated on Mac Conkey medium and blood agar. ${ }^{7}$ The inoculated specimens are incubated at $35 \pm 2{ }^{\circ} \mathrm{C}$ in ambient air overnight. Significant growth is deemed to be $\geq 10^{5}$ colony-forming units (CFU/mL). Identification of bacteria was performed by standard biochemical methods and morphology.

\section{Susceptibility testing}

Disc diffusion testing was performed by the KirbyBauer method. The results were interpreted according to the Clinical and Laboratory Standards Institute (CLSI) version M100-S21. ${ }^{7}$ Susceptibility testing for yeasts was not done in order to eliminate duplication.

The antibiotic discs used were for amikacin, cefotaxime, ceftazidime, cefuroxime, cotrimoxazole, imipenem, tazocin, ampicillin, cefoxtin, ceftriaxone, cephalothin, gentamicin, norfloxacin and meropenem. All 
the antimicrobials were placed after drying the plate for 3-5 min and incubated at $35 \pm 2{ }^{\circ} \mathrm{C}$ for 24 hours. Extended-spectrum $\beta$-lactamases (ESBLs) were interpreted by using zone diameter breakpoints.

Antibiotic disks of fosfomycin and nitrofurantoin, normally was not tested in Songklanagarind Laboratory, were added for this study and their inhibition zones recorded. Both fosfomycin (200 microgram) and nitrofurantoin (300 microgram) were obtained from the Oxoid Company.

The CLSI interpretation breakpoint for fosfomycin sensitivity is at least $16 \mathrm{~mm}$, intermediate is between 13-15 $\mathrm{mm}$ and resistant is at most $14 \mathrm{~mm}$. Nitrofurantoin sensitivity is at least $17 \mathrm{~mm}$, intermediate is between $15-16 \mathrm{~mm}$ and resistant is at most $14 \mathrm{~mm}^{7}$ Disc diffusion is required for fosfomycin Antimicrobial Susceptibility Testing (AST), and was performed according to the CLSI recommendations.

Reference strains of E. coli, ATCC 25922, and K. pneumoniae, ATCC 700603, were used as controls for the Gram-negative bacteria and were included in all daily runs.

\section{Data processing and analysis}

Discrete variables were expressed as percentages and proportions and compared using the chi-square test.

Not all organisms were tested against each antimicrobial agent, as the proportions of susceptible bacteria were calculated based on the number of strains actually tested for each antimicrobial agent. The bacterial susceptibilities were reported in terms of percentage and corresponding 95\% confidence interval $(\mathrm{Cl})$. The $95 \% \mathrm{Cl}$ were derived from exact binomial statistics calculation.

\section{Resulls}

The final group of subjects were 324 outpatients with UTIs and a positive uroculture. The characteristics of the study patients are shown in Table 1. There were 91 males and 233 females with a mean age of 59 years, with 88 uncomplicated UTIs (27.2\%) and 236 complicated UTIs (72.8\%). 29.9\% had been admitted to the hospital in the previous twelve months, and ceftriaxone was the most common antibiotic used (44.8\%).

The distribution of diameters of the disc diffusion inhibition zones, including fosfomycin and nitrofurantoin are shown in Figures 2 and 3.

Table 1 Patient demographics at enrollment

\begin{tabular}{|c|c|c|}
\hline Variable & Percentage & $95 \% \mathrm{Cl}$ \\
\hline \multicolumn{3}{|l|}{ Sex } \\
\hline Female & 71.9 & $66.7-76.7$ \\
\hline Male & 28.1 & 23.3-33.3 \\
\hline Mean age & 59.0 & $56.6-61.4$ \\
\hline \multicolumn{3}{|l|}{ OPD attended } \\
\hline Emergency Room & 58.6 & $53.1-64.1$ \\
\hline Internal Medicine \& Pediatrics & 15.4 & $11.7-19.8$ \\
\hline OB-GYN & 10.8 & $7.6-14.7$ \\
\hline Primary Care Unit & 8.1 & $5.3-11.5$ \\
\hline Uro-Surgery & 4.9 & $2.8-7.9$ \\
\hline Surgery \& Orthopedics & 2.2 & $0.9-4.4$ \\
\hline \multicolumn{3}{|l|}{ Classification of UTIs } \\
\hline Complicated & 72.8 & $67.6-77.6$ \\
\hline Uncomplicated & 27.2 & $24.2-34.2$ \\
\hline \multicolumn{3}{|l|}{ Antibiotic treatment } \\
\hline Ceftriaxone & 44.8 & $39.3-50.4$ \\
\hline Ofloxacin & 11.4 & $8.2-15.4$ \\
\hline Other & 43.8 & $38.3-49.4$ \\
\hline \multicolumn{3}{|l|}{ Urine collection method } \\
\hline Catheter & 36.4 & $31.2-41.9$ \\
\hline Midstream urine & 63.6 & $58.1-66.8$ \\
\hline $\begin{array}{l}\text { History of admission in last } 12 \\
\text { months }\end{array}$ & 29.9 & $56.0-61.0$ \\
\hline
\end{tabular}

$\mathrm{Cl}=$ confidence interval, OPD=Out Patient Department, OB-GYN= Obstetrics and Gynecology, UTIs=urinary tract infections 


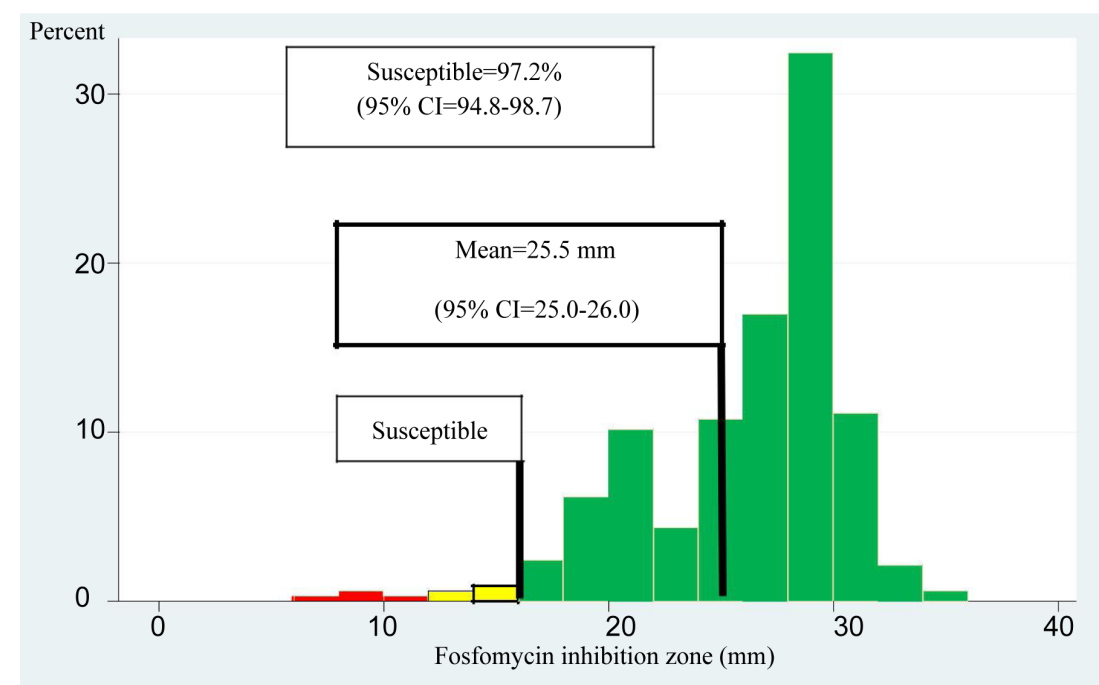

Figure 2 Graph showing the fosfomycin inhibition zones

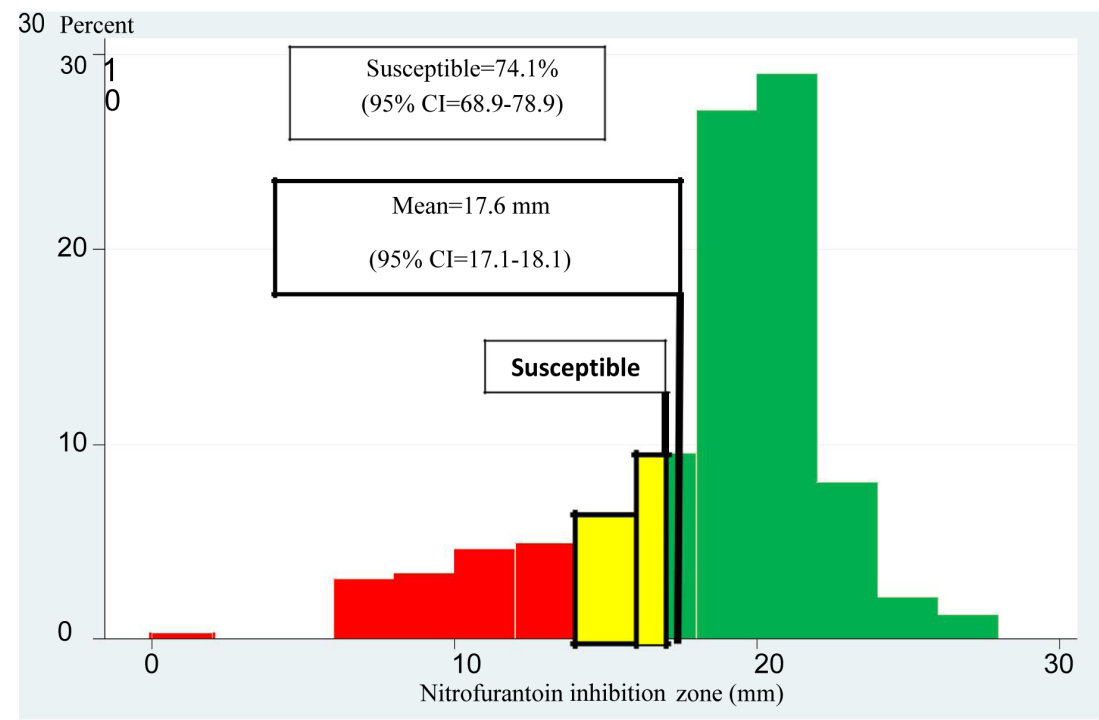

Figure 3 Graph showing the nitrofurantoin inhibition zones 
The most common factor associated with complicated UTIs in our study was male gender $(28.1 \%, 95 \% \mathrm{Cl}=23.2-$ 33.3), followed by diabetes mellitus (25.9\%, 95\% Cl=21.2$31.1)$ and history of urine catheter in the previous 12 months (15.7\%, 95\% Cl=23.2-33.3). The other most common factors were stone, benign prostatic hypertrophy and cancer of the urinary tract.

Table 2 shows that E. coli $(71.9 \%)$ was identified in 233 samples, ESBLs in $22.8 \%$ and non ESBLs in $49.1 \%$. The second most common microorganisms were $K$. pneumoniae $(16.7 \%)$, followed by others $(11.4 \%)$.

Table 2 Prevalence of uroculture pathogens

\begin{tabular}{lll}
\hline Bacteria & $\begin{array}{l}\text { Percent } \\
(\mathrm{N}=342)\end{array}$ & $95 \% \mathrm{Cl}$ \\
\hline Escherichia coli & 71.9 & $66.7-76.7$ \\
$\quad$ Non-ESBLs & 49.1 & $43.5-54.7$ \\
$\quad$ ESBLs & 22.8 & $18.4-27.8$ \\
Klebsiella pneumoniae & 16.7 & $12.8-21.2$ \\
$\quad$ Non-ESBLs & 8.0 & $5.3-11.5$ \\
$\quad$ ESBLs & 8.7 & $5.8-12.2$ \\
Enterobacter cloacae & 2.2 & $0.9-4.4$ \\
Enterococci & 2.2 & $0.9-4.4$ \\
Citrobacter koseri & 1.8 & $0.7-4.0$ \\
Proteus mirabilis & 1.2 & $0.3-3.1$ \\
Klebsiella oxytoca & 0.9 & $0.2-2.7$ \\
Serratia marcescens & 0.9 & $0.2-2.7$ \\
Citrobactera amalonaticus & 0.6 & $0.7-2.2$ \\
Others & 1.5 & $0.5-3.6$ \\
\hline
\end{tabular}

$\mathrm{Cl}=$ confidence interval, ESBLS=extended-spectrum $\beta$-lactamases Others: E. aerogenes, Pantoea agglomerans, K. ozaenae, P. vulgaris, Salmonella
Figure 2 shows that the susceptibility to fosfomycin of $E$. coli was $97.2 \%$. The average diameter of the inhibition zones was $25.5 \mathrm{~mm}$. The red, yellow, and green columns represent percentages of being resistant, intermediate, and susceptible, respectively.

Figure 3 shows that sensitivity results to nitrofurantoin, the susceptibility to nitrofurantoin of pathogens tested were $74.1 \%$. The average diameter of zones of inhibition was $17.6 \mathrm{~mm}$.

The susceptibility results to fosfomycin are shown in Table 3, which shows that E. coli had an overall susceptibility of $98.7 \%$; $97.3 \%$ in ESBLs and $99.4 \%$ in non ESBLs.

For nitrofurantoin, E. coli again had the highest susceptibility at $91.0 \%, 93.2 \%$ in ESBLs and $89.9 \%$ in non ESBLs. The second most common was others (40.5\%), followed with $K$. pneumoniae (24.1\%).

Table 4 shows that the total of 324 positive culture cases were classified into complicated $(72.8 \% \mathrm{n}=236)$ and uncomplicated $(27.2 \% \mathrm{n}=88)$ UTIs. In these categories, the susceptibilities to fosfomycin and nitrofurantoin were 97.0\% (95\% Cl=94.0-98.8) and 71.2\% (95\% Cl=64.9-76.9) in complicated UTIs and $97.7 \%(95 \% \mathrm{Cl}=92.0-99.7)$ and $81.8 \%(95 \% \mathrm{Cl}=72.1-89.2)$ in uncomplicated UTIS, respectively.

Table 5 shows the relationship between urine samples collected from the OPD with the highest number of UTIs cases and the susceptibility results to fosfomycin and nitrofurantoin. Overall, the susceptibility to fosfomycin was $>95.0 \%$ in all OPD cases. For nitrofurantoin the susceptibility was about 68.0-80.0\%, except in the OBGYN OPD which had a susceptibility of only $45.7 \%$.

Table 6 shows that the antibiotic with the highest bacterial non-susceptibility was ampicillin (80.8\%), but in that case there were only $2.7 \%$ non-susceptibility to fosfomycin and $29.0 \%$ to nitrofurantoin. The most commonly used antibiotic in this study was ceftriaxone, which had 
a bacterial non-susceptibility of $33.9 \%$, with $3.6 \%$ nonsusceptibility to fosfomycin. Overall, $50.0 \%$ of the samples were non-susceptible to norfloxacin, with overall $2.5 \%$ and
$24.7 \%$ non-susceptible to fosfomycin and nitrofurantoin, respectively.

Table 3 Susceptibility of various uroculture pathogens to fosfomycin and nitrofurantoin

\begin{tabular}{|c|c|c|c|c|c|}
\hline Bacteria & Number* & Fosfo $^{\S}(\%)$ & $95 \% \mathrm{Cl}$ & Nitro $^{\ddagger}(\%)$ & $95 \% \mathrm{Cl}$ \\
\hline Escherichia coli & 233 & 98.7 & $96.3-99.7$ & 91.0 & $86.6-94.3$ \\
\hline Non-ESBLs & 159 & 99.4 & $96.5-99.9$ & 89.9 & $84.2-94.1$ \\
\hline ESBLS & 74 & 97.3 & $90.6-99.7$ & 93.2 & $84.9-97.8$ \\
\hline Klebsiella pneumoniae & 54 & NA & NA & 24.1 & $13.5-37.6$ \\
\hline Non-ESBLs & 26 & NA & NA & 34.6 & $17.2-55.7$ \\
\hline ESBLS & 28 & NA & NA & 14.3 & $4.0-32.7$ \\
\hline Enterobacter cloacae & 7 & NA & NA & 14.3 & $3.6-57.9$ \\
\hline Enterococci & 7 & NA & NA & 85.7 & $42.1-99.6$ \\
\hline Citrobacter koseri & 6 & NA & NA & 33.3 & $4.3-77.7$ \\
\hline Proteus mirabilis & 4 & NA & NA & 0.0 & $0.0-60.2$ \\
\hline Klebsiella oxytoca & 3 & NA & NA & 66.7 & $9.4-99.2$ \\
\hline Serratia marcescens & 3 & NA & NA & 0.0 & $0.0-70.9$ \\
\hline Citrobacter amalonaticus & 2 & NA & NA & 0.0 & $0.0-84.2$ \\
\hline
\end{tabular}

*Number of strains tested, ${ }^{\S}$ Susceptible percentage to fosfomycin, ${ }^{\ddagger}$ Susceptible percentage to nitrofurantoin, NA=not available,

$\mathrm{Cl}=$ confidence interval, ESBLs=extended-spectrum $\beta$-lactamases

Table 4 Susceptibility of complicated and uncomplicated urinary tract infections (UTIS) to fosfomycin and nitrofurantoin

\begin{tabular}{llllc}
\hline Classification of UTIs & Fosfo $\left.{ }^{\S} \%\right)$ & $95 \% \mathrm{Cl}^{\dagger}$ & Nitro $(\%)$ & $95 \% \mathrm{Cl}^{\dagger}$ \\
\hline Complicated $\left(\mathrm{n}^{*}=236\right)$ & 97.0 & $94.0-98.8$ & 71.2 & $64.9-76.9$ \\
Uncomplicated $\left(\mathrm{n}^{*}=88\right)$ & 97.7 & $92.0-99.7$ & 81.8 & $72.1-89.2$ \\
\hline
\end{tabular}

*Number of strains tested, ${ }^{\circledR}$ Susceptible percentage to fosfomycin, ${ }^{\ddagger}$ Susceptible percentage to nitrofurantoin, ${ }^{\dagger} 95 \%$ confidence interval calculated by exact binomial statistics

$\mathrm{Cl}=$ confidence interval 
Table 5 Susceptibility of samples collected at the Out Patient Departments (OPD) to a certain dose of fosfomycin and nitrofurantoin

\begin{tabular}{lllllc}
\hline OPD & Number* & Fosfo $\left.^{\S} \%\right)$ & $95 \% \mathbf{C l}$ & Nitro $^{\ddagger}(\%)$ & $95 \% \mathrm{Cl}^{\dagger}$ \\
\hline Emergency Room & 190 & 95.7 & $91.9-98.2$ & 76.3 & $69.6-82.2$ \\
Medicine \& Pediatrics & 50 & 100.0 & $92.9-100.0$ & 82.0 & $68.6-91.4$ \\
OB-GYN & 35 & 97.1 & $85.1-99.9$ & 45.7 & $28.8-63.3$ \\
Primary Care Unit & 26 & 100.0 & $86.8-100.0$ & 80.8 & $68.6-91.4$ \\
Uro-Surgery & 16 & 100.0 & $79.4-100.0$ & 68.7 & $41.3-89.0$ \\
Surgery \& Orthopedics & 7 & 100.0 & $59.0-100.0$ & 85.7 & $42.1-99.6$ \\
\hline Total & 324 & 97.2 & $94.8-98.7$ & 74.1 & $68.9-78.8$ \\
\hline
\end{tabular}

${ }^{*}$ Number of strains tested, ${ }^{\$}$ Susceptible percentage to fosfomycin, ${ }^{\ddagger}$ Susceptible percentage to nitrofurantoin, ${ }^{\dagger} 95 \%$ confidence interval calculated by exact binomial statistics

$\mathrm{Cl}=$ confidence interval, OB-GYN=Obstetrics and Gynecology

Table 6 The non-susceptibility of various bacteria to standard antibiotics compared with their susceptibility to fosfomycin and nitrofurantoin

\begin{tabular}{llll}
\hline Antibiotic & $\begin{array}{l}\text { Non-susceptible } \\
(\%)\end{array}$ & $\begin{array}{l}\text { Non-susceptible to } \\
\text { fosfomycin (\%) }\end{array}$ & $\begin{array}{l}\text { Non-susceptible } \\
\text { tonitrofurantoin (\%) }\end{array}$ \\
\hline Ampicillin & 80.8 & 2.7 & 29.0 \\
Cephalothin & 68.2 & 2.7 & 26.2 \\
Cefuroxime & 61.1 & 2.5 & 26.3 \\
Norfloxacin & 50.0 & 2.5 & 24.7 \\
Cotrimoxazole & 48.5 & 2.5 & 29.3 \\
Cefotaxime & 34.6 & 3.6 & 30.3 \\
Ceftazidime & 34.3 & 3.6 & 29.7 \\
Ceftriaxone & 33.9 & 3.6 & 30.0 \\
Gentamicin & 19.1 & 3.2 & 27.4 \\
Cefoxitin & 17.0 & 3.6 & 43.6 \\
Sulperazon & 13.0 & 9.5 & 52.4 \\
Tazocin & 11.4 & 8.1 & 48.6 \\
\hline
\end{tabular}




\section{Discussion}

UTIs is one of the most common indications for prescribing antimicrobials to otherwise healthy communitydwelling women. The IDSA published a clinical practice guideline on the treatment of women with acute uncomplicated cystitis and pyelonephritis in 1999. ${ }^{1}$ Since then, antimicrobial resistance among uropathogens causing UTIs has increased. Therefore, much research is being done to look for other more effective antibiotics to replace the old ones. Given the wide resistance to older antibiotic first line drugs such as fluoroquinolones and trimethoprimsulfamethoxazole, doctors today are experimenting with alternative drugs such as fosfomycin and nitrofurantoin. Recently, The Inter-national Clinical Practice Guidelines for the Treatment of Acute Uncomplicated Cystitis and Pyelonephritis in Women: A 2010 Update by the Infectious Diseases Society of America and the European Society for Microbiology and Infectious Diseases, ${ }^{8}$ found that many pathogens resist even first-line drugs, thus fosfomycin and nitrofurantoin are used instead. Previously, our hospital did not have data concerning the susceptibility of major disease organisms to fosfomycin and nitrofurantoin, so this study was undertaken to provide us with data in order to help manage our patients and the drug-resistant bacteria appropriately.

In order to keep our data as free from possible confounders as possible, our sample included only patients with pyuria, and with a single type of organism identified, which would ensure that the pathogen was a uropathogen, and did not come from contamination. The total of 324 patients was the figure obtained from the binomial sample size formula, and also was limited by the available budget for data collection. Also, we collected data only from OPD and ER patients, which meant that we had no data from community-acquired UTIs inpatients, however, this was not considered a problem since most such patients have clinically severe disease which cannot be treated by the antibiotic treatments we were examining. ${ }^{8}$

Our study examined the distribution of microbial species causing UTIs in Songklanagarind Hospital during the year 2011, and their susceptibility patterns to the most commonly used oral and parenteral antimicrobial agents. We found that the two most common UTIs causative agents during that year were E. coli $(71.9 \%)$ and K. pneumoniae (16.7\%). Our findings are similar to other studies, although the prevalences vary. ${ }^{9-13}$

In this research, most cases were complicated UTIs $(72.8 \%)$, which was related to two factors, male gender $(28.1 \%)$ and diabetes mellitus $(25.9 \%)$. This research was performed in a tertiary care center where there was high pathogenic resistance, at $80.9 \%$ for ampicillin, $33.9 \%$ for ceftriaxone, $50.0 \%$ for norfloxacin, and $48.5 \%$ to cotrimoxazole, our first line drug, but all the pathogens studied were more than $70.0 \%$ susceptible to fosfomycin and nitrofurantoin so this research suggests treating UTIs with these agents.

Our drug susceptibility results were also categorized by different OPDs and the ER, and we found that the samples from all OPDs except OB-GYN were approximately equally susceptible to fosfomycin, while the susceptibilities to nitrofurantoin were quite variable in all of the OPD and the ER, ranging from $74.0-85.0 \%$, while again the OBGYN samples were much lower, at $45.7 \%$. We were not able to determine the reason for this difference, as there were only 35 samples from OB-GYN, which were not enough for analysis.

Because of the availability of these two drugs in Thailand, we have to consider the susceptibility of antimicrobial, their cost effectiveness, and the route of each drug. Nitrofurantoin for treatment of uncomplicated cystitis costs about 300 baht per course (100 mg bid for 5 days), which is cheaper than fosfomycin for the same condition 
which costs 406 baht (single dose), but it has low sensitivity and the patient must be treated orally which is not effective for severe cases with severe infection or in which the infection has extended to organs other than the urinary tract. Also, nitrofurantoin can have cross-reactions with other drugs, and has also been associated with pulmonary fibrosis.

Our study found that fosfomycin had higher efficacy for inhibition of uropathogens in outpatients of Songklanagarind Hospital. It can be administrated by either IV form and oral form so it can be used to treat severe cases, and it also doesn't have cross reactions with other drug. However, it is more expensive than nitrofurantoin and in oral form the drug has to be mixed with water first which may be difficult for the patient.

The study had one limitation that should be noted. It examined data from a tertiary care setting, so most of the patients were complicated UTIs, most notably with cancer, obstructive uropathy, or diabetes mellitus, which are difficult to treat. However, fortunately, male and diabetes mellitus are also common factors of complicated UTIs found in the other institutions, and therefore the study can be applied to those too. The merit of this study is that both urine culture and sensitivity analyses were performed, and consequently our results can be considered reliable.

\section{Conclusion}

The most common uropathogen in out patients with UTIs was E. coli (71.9\%), followed by K. pneumoniae (16.7\%). The susceptibility of $E$. coli to fosfomycin was $98.7 \%$, and the susceptibilities of E. coli and K. pneumoniae to nitrofurantoin were $91.0 \%$ and $24.1 \%$ respectively.

\section{References}

1. Warren JW, Abrutyn E, Hebel JR, Johnson JR, Schaeffer AJ, Stamm WE. Guidelines for antimicrobial treatment of uncomplicated acute bacterial cystitis and acute pyelonephritis in women. Clin Infect Dis 1999;29:745-58.
2. Sangsuwan T, Jamulitrat $S$. Antimicrobial resistance among urinary tract infection pathogens in the Outpatient Department of Songklanagarind Hospital in the year 2008 [Dissertation]. Songkhla: Prince of Songkla University; 2010.

3. Grude N, Potaturkina-Nesterova NI, Jenkins A, Strand L, Nowrouzian FL, Nyhus J, et al. A comparison of phylogenetic groups, virulence factors and antibiotic resistance in Russian and Norwegian isolates of Escherichia coli from urinary tract infection. Clin Microbiol Infect 2006;13:208-11.

4. Kahlmeter G, Poulsen H. Antimicrobial susceptibility of Escherichia coli from community-acquired urinary tract infections in Europe: the ECO.SENS study revisited. Int J Antimicrob Agents 2012;39:45-51.

5. Ferry SA, Holm SE, Stenlund H, Lundholm R, Monsen TJ. The natural course of uncom-plicated lower urinary tract infection in women illustrated by a randomized placebo controlled study. Scan J Infect Dis 2009;36:296-301.

6. Muvunyi CM, Masaisa F, Bayingana C, Mutesa L, Musemakweri A, Muhirwa G, et al. Decreased susceptibility to commonly used antimicrobial agents in bacterial pathogens isolated from urinary tract infections in Rwanda: need for new antimicrobial guidelines. Am J Trop Med Hyg 2011;84:923-8.

7. Clinical and Laboratory Standards Institute. Performance standards for antimicrobial susceptibility testing; twenty-first informational supplement; M100-S21. Wayne: The Institute; 2011.

8. Gupta K, Hooton TM, Naber KG, Wullt B, Colgan R, Miller LG, et al. International clinical practice guidelines for the treatment of acute uncomplicated cystitis and pyelonephritis in women: a 2010 updated by the Infectious Diseases Society of America and the European Society for Microbiology and Infectious Diseases. Clin Infect Dis 2011;52:103-20.

9. Kahlmeter G. Prevalence and antimicrobial susceptibility of pathogens in uncomplicated cystitis in Europe. The ECO.SEN study. Int J Antimicrob Agents 2003;22:49-52.

10. Raka L, Mulliqi-Osmani G, Berisha L, Begolli L, Omeragiq S, Parsons L, et al. Etiology and susceptibility of urinary tract isolates in Kosovo. Int J Antimicrob Agents 2004;23:2-5.

11. Zhanel GG, Hisanaga TL, Laing NM, DeCorby MR, Nichol KA, Palatnik LP, et al. Antimicrobial resistant in outpatient urinary isolates: final results from the North American Urinary Tract Infection Collaborative Alliance (NAUTICA). Int $\mathrm{J}$ Antimicrob Agents 2005;6:380-8. 
12. Nicole LE. Uncomplicated urinary tract infection in adults including uncomplicated pyelonephritis. Urol Clin North Am 2008;35:1-12.
13. Al-Tawfiq JA, Anani AA. Antimicrobial susceptibility pattern of bacterial pathogens causing urinary tract infections in a Saudi Arabian Hospital. Chemotherapy 2009;55:127-31. 